\title{
Los mapas interactivos, herramientas para la participación ciudadana
}

Recibido: 15 de agosto de 2018

Aceptado: 10 de octubre de 2018

\author{
Narcisa Medranda Morales \\ nmedranda@ups.edu.ec \\ Universidad Politécnica Salesiana (Ecuador) \\ Victoria Palacios Mieles \\ vdpalacios@puce.edu.ec \\ Pontificia Universidad Católica del Ecuador \\ Tomás Moromenacho Diaz \\ tdmoromenacho@puce.edu.ec \\ Pontificia Universidad Católica del Ecuador
}

Resumen: Los mapas interactivos son una herramienta digital que permite poner a disposición de las personas información de manera visual, en un solo conjunto. Además, ofrecen la flexibilidad necesaria para que cada persona pueda acceder a la información de su interés. Estas características convierten a estos mapas en una herramienta óptima para propiciar la participación ciudadana. Un ejemplo exitoso del uso de estos mapas (en tanto herramienta para fomentar la participación ciudadana) constituye el proyecto "Mapa Infoparticipa" el cual, a través del uso de estas herramientas, socializa los niveles de transparencia de los gobiernos locales en países como España, Ecuador, Colombia, Chile y Brasil. En este artículo se definen los mapas interactivos y se explica su constitución como herramienta para fomentar participación ciudadana, partiéndose de la experiencia del proyecto "Mapa Infoparticipa".

Palabras clave: Comunicación, mapas interactivos, participación ciudadana, web 3.0, Mapa Infoparticipa, democracia, interactividad, factor no-humano.

Abstract: Interactive maps are digital tools that make information available to people in a visual way, in a single set. In addition, they offer the necessary flexibility so that each person can access the information of their interest. These characteristics make these maps 
an optimal tool to promote citizen participation. A successful example of the use of these maps as a tool to encourage citizen participation is the Mapa Infoparticipa Project, which, with these tools, socializes the transparency levels of local governments in countries such as Spain, Ecuador, Colombia, Chile and Brazil. This article presents what interactive maps are and argues how they are a tool to encourage citizen participation, based on the experience of Mapa Infoparticipa Project.

Key words: Communication, Interactive Maps, Citizen Participation, Web 3.0, Infoparticipate Map, Democracy, Interactivity, Non-human Factor.

\section{Introducción}

Los mapas interactivos se han convertido en una herramienta que posibilita a las personas ejercer de mejor manera el control ciudadano respecto de las actuaciones de los representantes políticos que dirigen las instituciones públicas. Una de las características de esta herramienta es una mayor facilidad de acceso, pues la información está disponible en todo momento y en cualquier parte del mundo, lo que facilita hacer una auditoria cívica de la gestión pública. Las nuevas tecnologías de la información y comunicación (TIC), en tanto factor no-humano que permite la interacción en tiempo asincrónico, sostienen esta herramienta y la ponen al alcance de toda persona.

Los mapas interactivos humanizan al periodismo y democratizan la comunicación, ya que ofrecen la suficiente flexibilidad para que cada persona acceda a la información desde varios criterios de búsqueda y que esta se presente según sus necesidades y expectativas. El acceso, la democratización y la flexibilidad de la información se constituyen en características que hacen de los mapas interactivos una verdadera herramienta para el control ciudadano de las actuaciones del sector público.

El proyecto Mapa Infoparticipa es un ejemplo de mapa interactivo enfocado a generar una mayor implicación de la ciudadanía en la gestión de lo público, especialmente sobre los gobiernos locales, municipios y ayuntamientos. En el entorno digital de este proyecto se puede acceder a la información mediante varios criterios de búsqueda. Se puede buscar información sobre el alcalde, el partido político y otros miembros del gobierno. Asimismo, se pueden encontrar los porcentajes de transparencia en la gestión, entre otros parámetros bien establecidos.

Dadas estas características de esta herramienta y la experiencia de Mapa Infoparticipa, este artículo se plantea poner en evidencia que los mapas interactivos facilitan la ejecución, por parte de la ciudadanía, de la auditoria cívica sobre la gestión de los representantes políticos, generando una explicación de la gestión y de las prácticas comunicaciones de los representantes políticos. Sostenemos que los mapas interactivos facilitan el acceso a la información para la ciudadanía, mejoran las prácticas de los profesionales de la comunicación en el ámbito público y evidencian la gestión de los representantes políticos. 


\section{Mapas interactivos}

Son una herramienta digital que permite acceder a la información de manera dinámica, gráfica y selectiva. La construcción de contenido de esta herramienta puede realizarse de forma individual o colaborativa e irse ampliando y actualizando constantemente. Bosque y Zamora (2002), señalan que "la construcción y el uso del mapa debe tratarse como un todo, no sólo como un soporte de datos, sino también como un medio de comunicación de información territorial" (p. 62). Esta dimensión territorial es lo que caracteriza a esta herramienta y que la hace pertinente tanto para quienes buscan información específica sobre su localidad como para quienes requieren una visión geográfica más amplia sobre un determinado tema.

La propuesta de los mapas interactivos logra una mayor eficiencia en el mensaje publicado, dado que el ciudadano puede establecer su propia lógica para el manejo y análisis de la información que se presenta en la cartografía. En ese sentido, facilita el acceso a la información y la interacción (Medranda, 2017). De esta manera, es posible comprender de manera visual e interactuar sobre las situaciones representadas en los mapas (Bosque \& Zamora, 2002; Dorling \& Fairbairn, 1997). Los mapas facilitan por lo tanto el acceso, la interacción, la comprensión visual y territorial sobre un tema determinado.

Estos niveles de interacción han sido posibles gracias a las bondades de la web 3.0 que es el espacio donde confluyen los nuevos estadios de interacción social. En el ciberespacio se producen las nuevas formas de participación, investigación y comunicación; donde encontramos mapas con la ubicación exacta de los lugares en donde nos encontramos y herramientas para encontrar rutas óptimas para llegar más rápido a nuestro destino o buscar todo tipo de servicios.

Además, la web 3.0 unifica resultados de búsqueda para crear tendencias en los usuarios, es decir, automáticamente relaciona los términos más utilizados y personaliza la búsqueda del usuario; creando contenidos que estos están acostumbrados a ver y, por ende, genera vínculos de información con los contenidos más visitados. Lo particular de esta funcionalidad en Internet es la capacidad de vincular páginas con contenidos de distintos tipos y formatos. Cada vez existen más servicios de información que añaden datos provenientes de otras fuentes, con la finalidad de unificar las respuestas que ofrecen a los usuarios (Conexión ESAN, 2015). La web 3.0 ha permitido a las personas intercambiar contenidos sociales y que las personas puedan convertirse en prosumidores.

\subsection{La tecnología como actor no-humano y fundamento de los mapas interactivos}

Durante las últimas décadas, las TIC han ocasionado transformaciones y modificaciones profundas para la vida cotidiana a nivel global. Han contribuido con enfoques renovadores para estudios existentes y generando nuevos análisis para su propio entendimiento, desde puntos de vista multidisciplinarios. En ese proceso se ha incluido lo económico, lo psicológico, lo sociocultural y lo político. Se puede afirmar que la tecnología ha llegado a convertirse en un verdadero actor en la sociedad actual. 
Para los mapas interactivos, la tecnología es de fundamental importancia, ya que sirve como el ámbito adecuado y necesario para su implementación, desarrollo y utilización. Como lo analiza Latour, citado por Rosales (2014), "las tecnologías han dejado de ser consideradas como productos y se transforman en actores no humanos" (p. 68), es decir, empezaron a tomar un rol activo al lado de los actores humanos, en cuanto al ordenamiento y la transformación de la vida cotidiana. En esta línea, una persona como actor humano usa los mapas interactivos (actor no-humano), los cuales transforman y ordenan su vida cotidiana a todos los niveles, especialmente a nivel social y político.

A nivel político, los mapas interactivos constituyen una herramienta clave para transparentar la información. Estos niveles de transparencia estarán estrechamente relacionados con la calidad de información publicada. Sin embargo, gracias a la interactividad, la ciudadanía contará con herramientas para exigir mayor transparencia. Sólo así el ciudadano puede confiar en sus representantes y, consecuentemente, confiar en su información (Bertot, Jaeger \& Grimes, 2010; Grimmelikhuijsen \& Welch, 2012). Las posibilidades de acceso a la información y la transparencia ofrecida por los mapas interactivos muestran que es posible utilizarlos como un medio para que se cumplan los derechos y para que la información sea completamente accesible para toda persona (Medranda, 2017, p. 94).

\subsection{Los mapas interactivos como herramientas para la participación ciudadana}

Gracias a interactividad de la web 3.0 y el soporte de la tecnología (como actor no-humano), los mapas digitales han logrado el potencial de volverse cada vez más interactivos. Con esto logran ampliar las posibilidades de relación y acción entre medio digital, el sujeto y entre sujetos. Así, esta herramienta abre diversas posibilidades de aplicación y uso en la vida cotidiana de las personas y en las relaciones sociales.

Además, existen mapas interactivos que tienen otras formas y objetivos de utilización, especialmente en el ámbito sociopolítico. Se trata de una aplicación práctica que va más allá de encontrar direcciones u oferta de servicios y que logra llegar a un espacio sociopolítico, en tanto permite acceder a información pública. Al contar con información completa, el ciudadano puede juzgar las actuaciones de sus representantes políticos. De esta forma, se configura una herramienta que facilita la participación ciudadana y fomenta la democracia. Un ejemplo de esto es Mapa Infoparticipa (2018): es una iniciativa ciudadana que pretende brindar herramientas para mejorar la comunicación de las administraciones públicas y la participación ciudadana.

El Mapa Infoparticipa favorece la construcción de sociedades más humanas. Cabe indicar que ha sido acogido por las autoridades políticas y la ciudadanía en general. Dado que la demanda de esta herramienta ha sido alta, este proyecto está en la agenda política, mediática y ciudadana, así lo confirma Manfredi y Cabezuelo (2015).

El proyecto de transparencia ya está en la agenda política, mediática y ciudadana. Por eso, el desarrollo de un proyecto de investigación que vincule la comunicación pública local, la rendición de cuentas, la gestión y la participación resulta adecuado. 
Puede suponer un salto hacia delante en la medida que, mediante la información pública, los ciudadanos pueden participar en la vida pública con conocimiento e influir en su desarrollo, prevenir y controlar ineficiencias administrativas, corregir errores, denunciar arbitrariedades y profundizar en la convivencia local (p. 36).

Por otro lado, a través de los mapas interactivos, el periodismo tiene la capacidad de comunicar la gestión de los recursos económicos de un país (Beckett, 2010). Esto es posible gracias a la georreferenciación que presentan los mapas en la red y a la capacidad de recoger la información detallada y completa, actualizada e inteligible.

\section{Metodología del proyecto Mapa Infoparticipa}

Este proyecto surge en el contexto académico de la Universidad Autónoma de Barcelona y busca ofrecer información organizada y actualizada que permita a la ciudadanía realizar auditoria cívica de la transparencia y la calidad de la información que dan las administraciones públicas en sus páginas webs (Moreno, Molina, Corcoy, Aguilar \& Borràs, 2014). El proyecto ha considerado a las tecnologías digitales "como una herramienta útil para socializar los resultados de las evaluaciones de transparencia" (Medranda, 2017, p. 93). Por medio de los mapas, se proporciona información a la ciudadanía. Además, el Proyecto busca (en todo momento) facilitar y ampliar el acceso de la ciudadanía a la información.

El proyecto Mapa Infoparticipa busca democratizar y liberar la información de las actividades de los representantes políticos que han sido electos por votación popular, generando información de calidad que transparente la gestión de estos representantes y, de este modo, generar una cultura de auditoria cívica.

Para lograr esta meta, se han establecido una serie de indicadores, a partir de los cuales se evalúan las páginas web de las entidades públicas y se construyen los resultados. En la actualidad, el proceso consta de 53 indicadores, con lo cual se marca la casilla dentro del mapa si la información consta o se deja el casillero en blanco si no es posible encontrar dicha información. La información publicada hasta el momento corresponde a las evaluaciones de los diferentes municipios de Ecuador.

\section{Visualización de la información en el Mapa Infoparticipa}

Mediante su inserción en las páginas webs, los mapas interactivos en formato online permiten encontrar con simplicidad la información que se busca. Esta búsqueda se considera positiva si para llegar a su objetivo, un ciudadano no experto no supera los tres clics, lo que no es muy frecuente.

Los resultados de las evaluaciones forman una base de datos que se vincula a la representación gráfica de la cartografía. Cada institución evaluada se visualiza en fichas individuales que se georreferencian, traduciendo los resultados en colores: blanco para 
menos de 25\%; amarillo del 25 al 49\%; verde claro del 50\% al 74\%; y verde oscuro para el $100 \%$. De esta manera, al consultar la institución evaluada, los resultados se muestran con el color señalado en el infómetro.

Sobre la base de respuestas afirmativas, se determina finalmente el grado de transparencia en porcentajes y con una marcación a color. La muestra considerada en esta investigación fue de 106 municipios mayores de 25000 habitantes. Se aplicaron 53 indicadores con una guía de evaluación que determina cuando se marca o no un indicador como positivo. Cuando la información se encuentra en la web evaluada, en el mapa se marca el casillero. Si la información no consta, se deja en blanco. Esto permite que el infómetro registre la marcación y establezca el porcentaje de transparencia automáticamente.

Con los datos extraídos del infómetro, el Mapa Infoparticipa establece la entrega de un sello de calidad denominado "Sello Infoparticipa". Este premio es entregado a las instituciones que han obtenido el 99\% de transparencia en las evaluaciones de las webs.

\section{Resultados en Ecuador}

La metodología del Mapa Infoparticipa se aplica en Ecuador desde el año 2016 para la evaluación de las páginas web de los municipios. En las evaluaciones que se realizaron a los municipios ecuatorianos (figura 1), se determinó que 1 de 106 aprueba los índices. El municipio ecuatoriano que supera el $50 \%$ en los indicadores es Guayaquil. Cincuenta y seis (56) municipios de 106 obtuvieron entre 25\% y 49.99\%. Finalmente, 49 municipios de 106 no superaron el $25 \%$.

Figura 1: Niveles de transparencia.

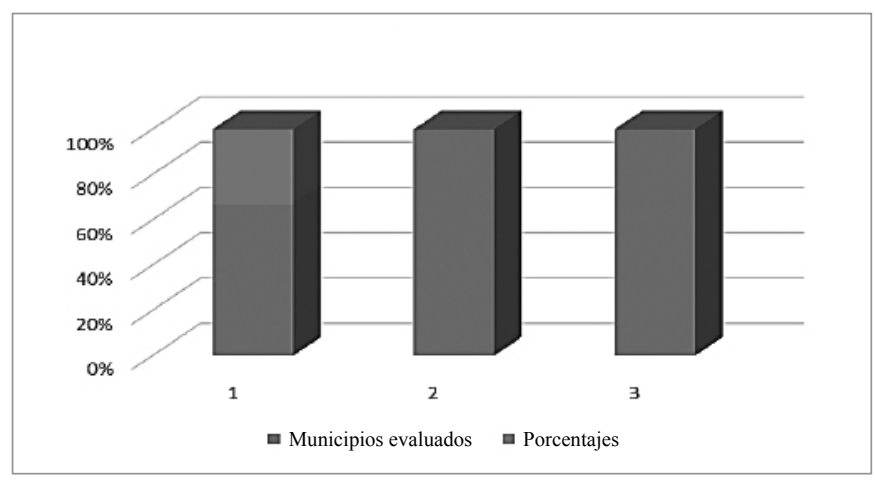

Fuente: Elaboración propia.

A continuación, se presenta la visualización de los resultados de los municipios ecuatorianos que fueron evaluados durante año 2017, mediante la herramienta de Mapa Infoparticipa. En la figura 1 se visualizan datos agrupados que permiten establecer los niveles de transparencia de los municipios de Ecuador evaluados. 
Figura 2: Visualización general de los municipios evaluados en Ecuador.

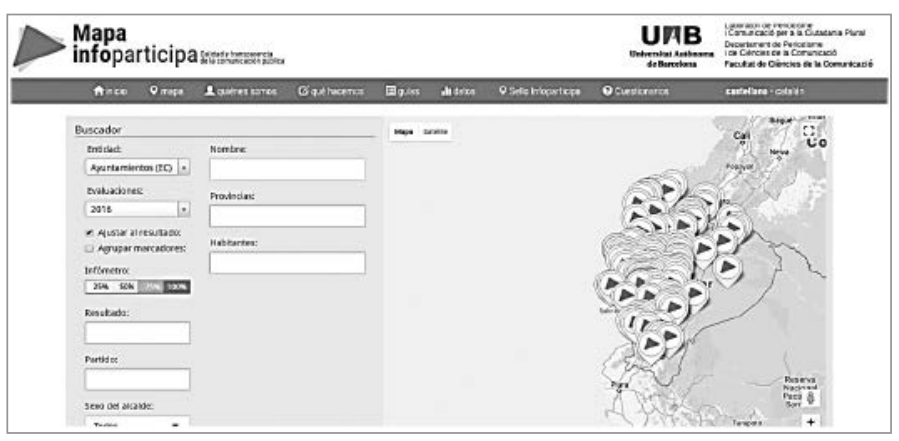

Fuente: Mapa Infoparticipa (2018).

Como se puede notar, la herramienta combina una serie de opciones de búsqueda con un mapa, donde se plasma la información deseada. Por medio de las herramientas de búsqueda, se visualizan los resultados según el porcentaje de cumplimiento de la ley, por sexo de los representantes, por partido político o por habitantes. La persona que busca información puede también navegar directamente sobre el mapa y gestionar la información desde una perspectiva territorial.

Figura 3: Visualización por porcentaje en el Mapa Infoparticipa.

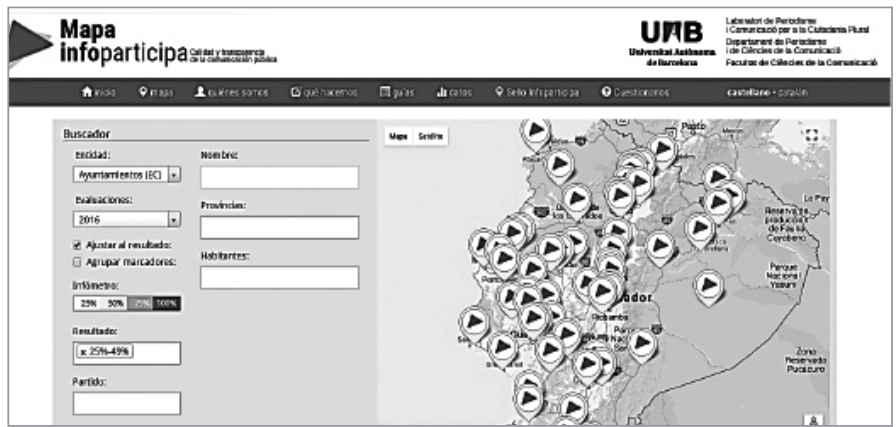

Fuente: Mapa Infoparticipa (2018).

En la figura 3 se visualiza únicamente aquellos municipios que han logrado entre un $25 \%$ y $49 \%$ en el cumplimiento de los índices de evaluación. La combinación de datos estadísticos y la localización geográfica amplía la comprensión de las personas sobre lo que sucede en la gestión pública y facilita una perspectiva general de la situación en el país.

La figura 4 ofrece información desde una perspectiva de género, mostrando municipios dirigidos por una mujer. En el mapa, más allá de los datos estadísticos, se puede identificar claramente cuál es la situación de los municipios en el país con respecto a sus alcaldes. Apenas seis municipios son dirigidos por mujeres. 
Figura 4: Visualización por sexo del representante político en el Mapa Infoparticipa.

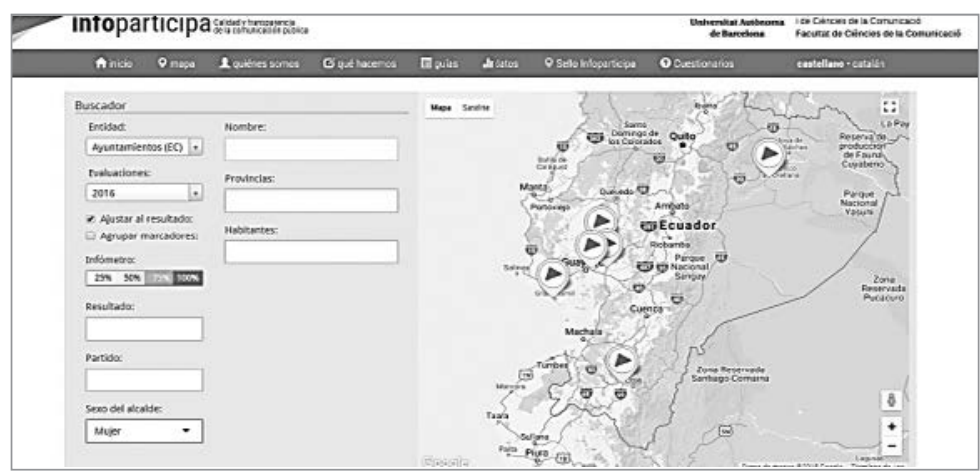

Fuente: Mapa Infoparticipa (2018).

Para mejorar el acceso a la información pública y/o posibilidades de análisis de los investigares, se pueden elegir otras variables de búsqueda, las cuales difieren según la selección de entidad. Al seleccionarse la entidad de los municipios ecuatorianos se cambian las variables específicas a número de habitantes, partido político y el sexo del alcalde.

De esta manera, los contenidos ofrecidos por el proyecto Mapa Infoparticipa ayudan a pensar en la participación ciudadana activa, ya que permiten el acceso libre y democrático a información tanto completa, contextualizada y con memoria como comprobable, contrastable y verificable por toda la ciudadanía mundial, resultando finalmente en la posibilitación de auditorías cívicas para el análisis crítico y reflexivo de las entidades examinadas. Por otro lado, la libre selección de las variables (básicas y específicas) garantizan la interactividad recíproca entre el mapa (actor no-humano) y el usuario (actor humano).

\section{Conclusiones}

En la actualidad, las sociedades democráticas están llamadas a incentivar la participación ciudadana, ya que este es un ejercicio que está relacionado con el ejercicio de derechos ciudadanos, con la posibilidad de acceder a la información generada en las instituciones y con la participación. Es por esto que los gobiernos deben poner a disposición de la ciudadanía una información oportuna, completa, contextualizada, contrastada, periodística y comprensible de sus acciones.

Mediante el acceso a la información pública, la ciudadanía puede conocer de primera mano las acciones de sus representantes políticos y, por lo tanto, hacer un seguimiento y control de la gestión de los políticos, con la finalidad de participar.

Los mapas interactivos brindan una herramienta didáctica que logra conectar los estudios tradicionales de la cartografía con los usos actuales de la web 3.0, promocionando tanto su 
rol de actor activo no-humano como la democratización general de información, lográndose (en el caso de Mapa Infoparticipa) objetivos pragmáticos, tales como transparencia, vigilancia de la política y de los medios de comunicación. Esto provoca mayores niveles de participación ciudadana y fortalecen la democrática a nivel global.

Actualmente, la Internet juega el papel un actor político no-humano. Su expansión ha llegado a tal nivel que es posible afirmar que envuelve la vida cotidiana de las personas, pero también la política y la pública. La web 3.0 ofrece la capacidad de navegación personalizada para el usuario, así como facilidades de ubicación y resolución de problemas al instante. Asimismo, amplía el espectro de la información, más allá de lo que presentan los medios de comunicación tradicionales, algunos de los cuales, actualmente se han integrado a la web para tener mayor alcance.

\section{Referencias}

Albornoz, M. \& Rosales, R. (2012). Periodismo ciudadano y Twitter. El caso del 30-S ecuatoriano. Revista Versión Estudios de Comunicación Política. Nueva Época, 30, 91-101. Recuperado de http://www.flacsoandes.edu.ec/sites/default/ files/\%25f/agora/files/periodismo_ciudadano_y_twitter.pdf

Beckett, C. (2010). Más cerca del ciudadano activo. El rescate del periodismo. Infoamérica. Iberoamerican Communication Review, 2, 45-57. Recuperado de https://www. infoamerica.org/icr/n02/beckett.pdf

Bertot, J., Jaeger, P. \& Grimes, J. (2010). Using ICTs to create a culture of transparency E-government and social media as openness and anti-corruption tools for societies. Government Information Quarterly, 27(3), 264-271. Recuperado de http://www. sciencedirect.com/science/article/pii/S0740624X10000201\#

Bosque, S. \& Zamora L. (2002). Visualización geográfica y nuevas cartografías. Revista Internacional de Ciencia y tecnología de la Información Geográfica, 2, 61-77. Recuperado de http://geofocus.rediris.es/docPDF/Articulo4_2002.pdf

Conexión ESAN (29 de mayo de 2015). Web 3.0: diez características que te permitirán identificarla. Recuperado de https://www.esan.edu.pe/apuntesempresariales/2015/05/web-3-diez-caracteristicas-que-te-permitiranidentificarla/

Dorling, D. \& Fairbairn, D. (1997). Mapping ways of representing the World. Londres: Addison Wesley Logman.

Ferré, C. (Ed.) (2014). El uso de las redes sociales: ciudadanía, política y comunicación. La investigación en España y Brasil. Barcelona: UAB. Recuperado de http:// incom.uab.cat/download/eBook_6_InComUAB_redessociales.pdf 
Grimmelikhuijsen, S. \& Welch, E. (2012). Developing and testing a theoretical framework for computermediated transparency of local governments. Public Administration Review, 7(4), 562-571. doi: 10.1111/j.1540-6210.2011.02532.x

Información Transparente (2010). El derecho a la información. Recuperado de https:// informaciontransparente.wordpress.com/tag/ecuador/.

Kaufmann, D. \& Bellver, A. (2005). Transparenting Transparency: Initial Empirics and Policy Applications. Recuperado de http://siteresources.worldbank.org/ INTEAPREGTOPRURDEV/Resources/573691-1175901454225/seminar 1 background_reading.pdf

Lindstedt, C. \& Naurin, D. (2010). Transparency is not enough: Making transparency effective in reducing corruption. International Political Science Review, 31(3), 301322. doi: $10.1177 / 0192512110377602$

López, P. \& Medranda, N. (2016). Transparencia, comunicación institucional e información pública en Ecuador: desarrollo metodológico y análisis de las prefecturas de Pichincha, Guayas e Imbabura. Actas del II Simposio de la Red Internacional de Investigación de Gestión de la Comunicación. Quito, Ecuador.

Manfredi, J. (2010). Periodismo y transparencia informativa. Cuadernos de periodistas. Revista de la Asociación de la Prensa de Madrid, 19, 113-123. Recuperado de https://dialnet.unirioja.es/servlet/articulo?codigo $=5255317$

Manfredi, J. \& Cabezuelo, F. (2015). Innovación periodística para la rendición de cuentas. En P. Molina (Coord.), Transparencia de la comunicación pública local. El Mapa Infoparticipa (www.mapainfoparticipa.com) (pp. 35-46). Recuperado de http:// www.cuadernosartesanos.org/2015/cac78.pdf

Mapa Inforparticipa (2018). Inicio: ¿Quiénes somos? Recuperado de http://www. mapainfoparticipa.com/index/home/5

Medranda, N. (2015). Elementos legales para la evaluación de transparencia en Ecuador. En P. Molina (Coord.), Transparencia de la comunicación pública local. El Mapa Infoparticipa (www.mapainfoparticipa.com) (pp. 197-209). Recuperado de http:// www.cuadernosartesanos.org/2015/cac78.pdf

Medranda, N. (2017). Calidad y transparencia en la información y comunicación que se emite a través de las páginas webs de los Municipios: Comparación de caso Ecuador y España (tesis doctoral). Universidad Autónoma de Barcelona, España. 
Moreno, A., Molina, P., Corcoy, M., Aguilar, A. \& Borràs, M. (2013). Infoparticip@: periodismo para la participación ciudadana en el control democrático. Criterios, metodologías y herramientas. Estudios sobre el mensaje periodístico, 19(2), 783803. doi: 10.5209/rev_ESMP.2013.v19.n2.43471

Rosales, R. (2014). Dinámicas de identidad y representación en la cultura gamer ecuatoriana. Chasqui, 125, 67-75. Recuperado de http:/www.revistachasqui.org/ index.php/chasqui/article/view/43/55 